\title{
Warum ein Heft zur Grossgruppenarbeit?
}

\section{Manfred Gellert • Falko von Ameln}

Wer einen großen Personenkreis und verschiedene Interessengruppen in einer lebendigen Form informieren, an Planungen und Veränderungsprozessen beteiligen und gleichzeitig in einen strukturierten Austausch bringen möchte, dem bieten Großgruppenmethoden ein breites Spektrum. Mit Großgruppenkonferenzen sind nicht einfach Versammlungen mit vielen Menschen gemeint, sondern der geplante und strukturierte Einbezug möglichst aller Betroffenen in ein gemeinsames Themenfeld. Von daher werden diese Ansätze in den unterschiedlichsten Organisationen und zu unterschiedlichsten Themen eingesetzt, das reicht von der partizipativen Gestaltung von Planungsprozessen im Rahmen der Stadtentwicklung über Zukunftskonferenzen von großen Unternehmen bis hin zur lebendigen Gestaltung von Anfangsphasen in großen Kongressen.

Beim Blick durch die Fachliteratur drängt sich der Verdacht auf, dass bisher überwiegend die „Systemiker“ und Organisationsberater das Feld der Arbeit mit großen Gruppen besetzt haben. Techniken wie „Open Space“, „World Cafe“, „Appreciative Inquiry“ oder „Zukunftskonferenz" finden sich in Beschreibungen von Weiterbildungsangeboten verschiedener Institute oder in Prozessberichten von Veranstaltungen immer wieder.

Wo sind eigentlich die Psychodramatiker mit ihren Ansätzen wie Soziodrama, Organisationsaufstellungsarbeit (bewusst nicht nach Hellinger und Jüngern) sondern im Ursprung nach Morenos Prinzipien, oder auch den Möglichkeiten der Soziometrie, speziell der Aktionssoziometrie, geblieben? Eine Ausnahme in der Fachliteratur bildet eigentlich nur das von Thomas Wittinger herausgegebene Handbuch zum Soziodrama in dem verschiedene Soziodramakonzepte in unterschiedlichen Anwendungsfeldern aufgezeigt werden und in dem gleichzeitig ein Beitrag zur Theoriebildung des Soziodramas vorgenommen wird.

Müssen wir uns eigentlich verstecken oder haben wir zu der Thematik nichts Substantielles beizutragen?

\footnotetext{
Online publiziert: 01.09 .2009

(C) VS-Verlag 2009

Dr. M. Gellert $(\square)$

Heinrich-Mahla-Str. 46, 63571 Gelnhausen, Deutschland

E-Mail: ConGel@t-online.de

www.concepta-team.de

F. von Ameln

Am Diekschloot 11b, 26506 Norden, Deutschland

E-Mail: info@vonameln.net
} 
Wir meinen nein, denn unsere Ansätze sind außerordentlich hilfreich und erweiternd in der Arbeit mit großen Gruppen.

Das vorliegende Themenheft versucht hier ein wenig die Lücke zu füllen, um verschiedene psychodramatische/soziometrische Ansätze als Beitrag zur Arbeit mit großen Gruppen in möglichst großer Breite vorzustellen und kritisch zu reflektieren.

Nach dem einleitenden Artikel von Jörg Burmeister haben wir die Artikel im Heft vier Überschriften zugeordnet:

Soziodrama/Soziometrie, Aufstellungsarbeit, Theaterarbeit und „Der andere Artikel“.

Jörg Burmeisters einleitender Artikel versteht sich als theoretisch-konzeptuelle Grundlegung für die Arbeit mit großen Gruppen. Er fasst Theoriebildung, massgebliche Konzepte sowie methodische Implikationen der Grossgruppenarbeit zusammen. Nach einem kurzen Abriss psychoanalytischer Konzepte zeigt Burmeister auf, wie sich eine Theorie der Grossgruppe aus psychodramatischer Perspektive anlegen lässt. Ein besonderer Schwerpunkt liegt dabei auf den Konzepten der Kultur und des Co-Unbewussten. Burmeisters Beitrag schließt mit praxisorientierten Hinweisen für die handlungsorientierte Arbeit in Grossgruppen sowie zu Gefahren, Risiken und Vorsichtsmaßnahmen, die man dabei berücksichtigen sollte.

\section{Soziodrama/Soziometrie}

Hilde Gött greift in ihrem Artikel „Konfrontation mit den Folgen von Auschwitz - Ein Soziodrama mit Großgruppen" ein sehr emotional besetztes Thema auf, indem sie ihren Ansatz des Soziodramas in Workshops beschreibt, die sie zusammen mit Yaacov Naor durchführt.

Ziel ist die Auseinandersetzung und Begegnung mit Spuren des Holocausts in der Gegenwart. Sie untersuchen die verschiedenen Arten der Internalisierung von Erfahrungen in den Rollen der Täter und der Opfer während der NS-Zeit und ermöglichen damit die Begegnung mit der Gegenseite. Nach ihrer Aussage wird damit keine Versöhnung oder Vergebung angestrebt, obwohl diese der Erfahrung nach von manchen Nachkommen aus Täterfamilien erhofft wird. Ihr Ziel ist es, einige Wunden bei den Teilnehmenden zu heilen und einen Dialog zu eröffnen.

Fred Dorn und Gotlind Kasper stellen in ihrem Beitrag: „Gewaltprävention und Umgang mit Gewalt an Schulen - Soziodrama in der Ausbildung für Lehrkräfte im Vorbereitungsdienst" die Vorbereitung, Durchführung und Auswertungsschritte eines Soziodramas vor. Dieser Workshop findet jährlich innerhalb der Referendarsausbildung in Hessen im Rahmen eines Seminars mit dem Thema: „Umgang mit Gewalt an Schulen“ statt. Nach einer Darstellung der thematischen Relevanz folgt eine Auseinandersetzung mit Gewalt als einem institutionellen wie auch gesellschaftlichen Phänomen. Danach folgt die Darlegung der Chancen, Zielen und Lernfeldern im methodischen, also soziodramatischen wie auch inhaltlichem Kontext.

In einem Gespräch mit Sabine Spitzer berichtet Helmut Haselbacher über seine soziodramatische Arbeit mit großen Gruppen. Ausgehend von Haselbachers Arbeit auf dem 
27. Psychodrama-Symposion in Spital am Phyrn (Österreich) zum Thema „Abhängigkeiten“" entwickelt sich ein anregendes Gespräch, das nicht nur interessante Einblicke in die Praxis, sondern auch Inspiration für Soziodramatiker/innen (und solche die es werden wollen) vermittelt.

Niko Schad und Raimund Gebhardt stellen eine Outdoor-Großgruppenaktion vor. Sie interpretieren das Geschehen zunächst von einem erlebnispädagogischen und dann von einem psychodramatisch-soziodramatischen Standpunkt aus. Auf einer Ebene gibt ihr Artikel somit einen interessanten Einblick in eine kreative Variante der Großgruppenarbeit. Auf einer weiteren Ebene leistet er aber auch einen Beitrag zur schulenübergreifenden Verständigung, indem er die Parallelen, aber auch Unterschiede zwischen verschiedenen eng verwandten handlungsorientierten Verfahren darstellt.

Julia Pischetsrieder stellt in Ihrem Artikel: „Organisationsentwicklung durch regelmäßige Großgruppen-Zielkonferenzen, Individual und Teamcoaching-Prozesse - ein Fallbeispiel" vor, wie sich Mitarbeiter und Mitarbeiterinnen eines Unternehmens in die Unternehmensentwicklung wirkungsvoll durch regelmäßige Zielkonferenzen integrieren lassen. Bewährt hat sich dabei die gleichzeitige Verknüpfung mit Maßnahmen wie z.B. Individualcoaching und/oder Teamcoaching im Rahmen eines Organisationsentwicklungsprozesses. In dem Beitrag wird die gewählte Vorgehensweise im Kontext eines Unternehmens als Fallbeispiel dargestellt - Rahmenbedingung, Voraussetzung und Dramaturgieelemente sowie sich daraus ergebende Erkenntnisse werden beschrieben.

\section{Aufstellungsarbeit}

Roswitha Riepl beschreibt in ihrem Artikel: „Politische Konfliktberatung mit soziodramatischer Aufstellungsarbeit" das Zusammenspiel von Soziodrama, soziometrischer Aufstellungsarbeit und politischer Konfliktberatung in der Arbeit mit größeren Gruppen am Beispiel des sogenannten „Ortstafelkonflikts“ im südösterreichischen Bundesland Kärnten. Ziel dieser methodischen Verschränkung der drei erwähnten Ansätze ist es, konkrete gesellschaftspolitische Konflikte auf ihre unterschwelligen Konflikt-Konstellationen und auf ihre oberschwelligen Konflikt-Dramen hin zu untersuchen. In einem dargestellten zweiten Praxisbeispiel berichtet sie über eine im Fernsehen von ihr und anderen Psychodramatikerinnen angeleitete soziodramatische Aufstellungsarbeit zum Kräfteverhältnis der politischen Parlamentsparteien in Österreich.

Ilona Ebbers und Reinhard Schulte befassen sich in ihrem Beitrag: „Organisationsaufstellungen als Beratungskonzept für Gründerpersonen“ mit der Versuch ein Beratungskonzept für Existenzgründerinnen und - gründer zu entwickeln. Nach der theoretischen Schilderung des Referenzrahmens der Methode wird das Ablaufschema einer Organisationsaufstellung präsentiert mit anschließender Darstellung eines Fallbeispiels aus der Praxis der Autoren. Der Artikel zeigt auf, wie Organisationsaufstellungen eine wertvolle Erweiterung des Beratungs- und Coachingspektrums für werdende Selbständige bieten können. 


\section{Theaterarbeit}

Michael Wrentschur würdigt in seinem Nachruf das Lebenswerk Augusto Boals, der am 2. Mai 2009 verstorben ist.

Boals Theateransätze, vor allem aus dem Theater der Unterdrückten, die in der Arbeit mit großen Gruppen vor allem in der politischen Arbeit in Lateinamerika Eingang gefunden haben, stehen in unmittelbarer Nachbarschaft mit Soziodramaansätzen und lassen sich in der Praxis der Arbeit mit großen Gruppen hervorragend verbinden. Auf Unterschiede und Gemeinsamkeiten hat Daniel Feldhendler an anderer Stelle hingewiesen

Johana Prawitasari-Hadiyono und ihre Kolleginnen berichten über die Arbeit mit Erdbebenopfern in Zentraljava (Indonesien). Ihr Konzept steht dem Theater methodisch näher als dem Soziodrama, verfolgt jedoch einen soziodramatisch-gruppentherapeutischen Anspruch, wie er bereits in Morenos soziodramatischer Arbeit angelegt ist. Zum einen bezieht Prawitasari-Hadiyonos Projekt die gesamte Dorfgemeinschaft in einen mehrmonatigen soziotherapeutischen Prozess ein, in dessen Folge das traumatisierende Erlebnis und seine Folgen aufgearbeitet werden. Zum anderen thematisiert das Projekt nicht nur die Ebene der individuellen und kollektiven Emotion, sondern auch die gesellschaftspolitischen Kontextbedingungen der nach auf das Erdbeben folgenden Katastrophenhilfe. Gleichzeitig knüpft das Projekt an tradierte Mechanismen der sozialen Integration an und trägt so zur Stärkung der lokalen Kultur bei. Das englische Original dieses Artikels kann in der Online-Version der Zeitschrift (www.zps-digital.de) abgerufen werden.

\section{4. „Der andere Artikel“}

Matthias Spörrle, Maria Strobel und Christian Stadler erforschen in ihrer empirischen Studie die Zusammenhänge zwischen Kulturzugehörigkeit, Größe und Dichte von sozialen Netzwerken und Lebenszufriedenheit. Ihr Beitrag ist ein wichtiger Vorstoß auf ein für die psychodramatische Arbeit wichtiges, aber bislang kaum erforschtes Terrain. Angesichts der differenzierten Ergebnisse aus dem Vergleich der Daten aus Deutschland/Österreich, Israel und Nigeria warnen die Autoren davor, in der therapeutischen Arbeit aus Netzwerkdaten voreilige Schlüsse zu ziehen (z.B. ,zahlenmäßig kleines soziales Netzwerk bedeutet grundsätzlich ein großes Problem“). Der Artikel schließt mit dem Plädoyer, soziale Netzwerke in ihren Qualitäten stärker in Beratung und Therapie einzubeziehen. 